\title{
MicroRNA-663b targets GAB2 to restrict cell proliferation and invasion in hepatocellular carcinoma
}

\author{
LIPING GUO $^{1}$, BAOLI LI ${ }^{2}$, MEIJING MIAO ${ }^{3}$, JIANJUN YANG $^{4}$ and JINSHAN JI ${ }^{5}$ \\ Departments of ${ }^{1}$ Pathology, ${ }^{2}$ Pharmacology and ${ }^{3}$ Nursing, Medical College of Yan'an University; \\ ${ }^{4}$ Department of Interventional Radiology, Affiliated Hospital of Yan'an University; ${ }^{5}$ Department of Preventive Medicine, \\ Medical College of Yan'an University, Yan'an, Shanxi 716000, P.R. China
}

Received May 12, 2018; Accepted November 28, 2018

DOI: $10.3892 / \mathrm{mmr} .2019 .9934$

\begin{abstract}
Previous studies have demonstrated that numerous tumor-specific microRNAs (miRNAs) are upregulated or downregulated in hepatocellular carcinoma (HCC), and that their dysregulation is implicated in HCC occurrence and development. Therefore, investigation of crucial miRNAs involved in $\mathrm{HCC}$ oncogenesis and progression may provide novel insights into the therapy of patients with this malignant tumor. In the present study, reverse transcription-quantitative polymerase chain reaction (RT-qPCR) assays were performed to detect tissue and cellular expression levels of miRNA-663b (miR-663b) in HCC. The effects of miR-663b overexpression on the proliferation and invasion of HCC cells were examined using Cell Counting Kit-8 and Transwell invasion assays, respectively. The direct target of miR-663b in HCC cells was determined by bioinformatics analysis, luciferase reporter assay, RT-qPCR and western blot analysis. It was observed that miR-663b was expressed at low levels in HCC tissues and cell lines. miR-663b upregulation suppressed the proliferative and invasive abilities of HCC cells. Additionally, Grb2-associated binding 2 (GAB2) was regarded as a direct target gene of miR-663b in HCC cells. Furthermore, GAB2 was overexpressed in $\mathrm{HCC}$ tissues, and overexpression of GAB2 was inversely correlated with levels of miR-663b. GAB2 overexpression was able to rescue the suppressive effects of miR-663b on HCC cells. These results demonstrated that this newly-identified miR-663b/GAB2 axis may be implicated in HCC occurrence and development.
\end{abstract}

Correspondence to: Professor Liping Guo, Department of Pathology, Medical College of Yan'an University, 38 Guanghua Road, Yan'an, Shanxi 716000, P.R. China

E-mail: yxyglp@126.com

Key words: hepatocellular carcinoma, microRNA-663b, Grb2-associated binding 2, proliferation, invasion

\section{Introduction}

Hepatocellular carcinoma (HCC), the most common malignant tumor of the liver, is the fourth most prevalent cancer and the third leading cause of cancer-associated mortality in the world (1). It is estimated that there is $\sim 792,000$ incident cases and 818,000 mortalities due to HCC each year worldwide (2). Despite the continuous progresses made in diagnosis and therapy, the long-term survival of patients with HCC remains poor, with a 5 -year survival rate of $\sim 30 \%(3,4)$. The poor prognosis of patients with $\mathrm{HCC}$ is primarily due to high rate of intrahepatic and distal metastasis, recurrence and the lack of effective therapeutic methods for those patients diagnosed at advanced stages $(5,6)$. Several well-documented factors have been identified to be closely associated with HCC initiation and progression, including chronic infection with hepatitis $\mathrm{B}$ or $\mathrm{C}$ virus, alcoholism, aflatoxin and excessive obesity caused by high-fat diets $(7,8)$; however, the detailed mechanisms underlying the pathogenesis of HCC remain poorly characterized. Therefore, it is imperative to improve the understanding of the mechanisms involved in HCC occurrence and development, which may contribute to the identification of potential effective therapeutic options for the treatment of patients with this disease.

MicroRNAs (miRNAs) are a group of endogenous, non-coding and short regulatory RNA molecules that are 18-24 nucleotides in length (9). miRNAs bind to the 3'-untranslated regions (UTRs) of their target genes to decrease their translation and/or promote mRNAs degradation (10). miRNAs are differently expressed in a number of types of human cancer, including HCC (11), and ovarian (12), thyroid (13) and gastric cancer (14). Dysregulated miRNAs serve a pivotal role in numerous biological behaviors, including cell proliferation, cell cycle, apoptosis, metastasis and angiogenesis (15-17). miRNAs are able to serve tumor-suppressing or oncogenic roles in the formation and progression of $\mathrm{HCC}$, which primarily depends on the characteristic of their target genes $(18,19)$. For example, miR-29a inhibits HCC cell proliferation, colony formation and inhibits cell cycle progression by directly targeting Sirtuin 1 (15). miR-1468 is upregulated in HCC and promotes aggressive tumor behaviors by activating peroxisome proliferator-activated receptor gamma-mediated protein B kinase signaling (20). Considering their crucial roles 
in HCC, miRNAs have the potential to be developed as novel biomarkers for diagnosis and therapeutic targets for treating patients with HCC.

Abnormal miR-663b expression has been previously described in different types of human cancer, including nasopharyngeal carcinoma (21), bladder cancer (22), osteosarcoma (23) and endometrial cancer (24). However, the expression pattern, potential biological roles and underlying mechanisms of miR-663b in HCC remain largely unknown. Therefore, the present study aimed to detect miR-663b expression in HCC tissues and cell lines, determine its effects and investigate the molecular regulatory mechanism of miR-663b in HCC progression. To the best of our knowledge, these data indicate for the first time a fundamental role for miR-663b in the pathogenesis of HCC.

\section{Materials and methods}

Patients and tissue specimens. In total, 34 pairs of HCC tissues and adjacent normal tissues were obtained from patients (23 males, 11 females; age range 43-72 years; median age, 61 years) who underwent surgical resection at the Affiliated Hospital of Yan'an University (Yan'an, China) between May 2015 and February 2017. Adjacent normal tissues were obtained $2 \mathrm{~cm}$ away from HCC tissues. The clinicopathological features of these patients are summarized in Table I. Patients were diagnosed using the tumor-node-metastasis (TNM) system (25). Patients treated with radiotherapy, chemotherapy or other treatments prior to surgery were excluded from the study cohort.

The present study was approved by the Ethics Committee of Affiliated Hospital of Yan'an University. Written informed consent was obtained from all participants.

Cell lines and culture conditions. A total of 2 human HCC Huh7 and Hep3B cell lines and the immortalized normal human liver epithelial L-O2 cell line were purchased from Institute of Biochemistry and Cell Biology, Shanghai Institutes for Biological Sciences, Chinese Academy of Science (Shanghai, China). All cells were grown at $37^{\circ} \mathrm{C}$ in a humidified atmosphere containing $5 \% \mathrm{CO}_{2}$ and $95 \%$ air, and cultured in Dulbecco's modified Eagle medium (DMEM) containing $10 \%$ fetal bovine serum (FBS), $100 \mu / \mathrm{ml}$ penicillin and $100 \mu \mathrm{g} / \mathrm{ml}$ streptomycin (all from Gibco; Thermo Fisher Scientific, Inc., Waltham, MA, USA).

Oligonucleotide transfection. Cells were plated into 6-well plates with a density of $6 \times 10^{5}$ cells/well. Then, $12 \mathrm{~h}$ after inoculation, cells were transfected with miRNA negative control mimics (miR-NC) or miR-663b mimics (both from Guangzhou Ribobio Co., Ltd., Guangzhou, China) using Lipofectamine ${ }^{\circledR} 2000$ (Invitrogen; Thermo Fisher Scientific, Inc.), as per the manufacturer's protocol. To restore Grb2-associated binding 2 (GAB2) expression, the full length sequence of GAB2 was chemically synthesized by Guangzhou Fueneng Gene Co., Ltd. (Guangzhou, China), cloned into pcDNA3.1 vector and named as pcDNA3.1-GAB2. The transfection efficiencies of miR-663b mimics and pcDNA3.1-GAB2 were determined using reverse transcription-quantitative polymerase chain reaction (RT-qPCR) and western blot analysis, respectively. RT-qPCR and western blot analysis were performed at 48 and $72 \mathrm{~h}$ after transfection, and carried out as described in the following paragraphs. Cell Counting kit-8 (CCK-8) and Transwell invasion assays were conducted at 24 and $48 \mathrm{~h}$ post-transfection, respectively.

$R N A$ extraction and RT-qPCR. TRIzol ${ }^{\circledR}$ reagent (Thermo Fisher Scientific, Inc.) was used to isolate total RNA from tissue samples or cells. The concentration of total RNA was measured using a NanoDrop 1000 spectrophotometer (NanoDrop Technologies; Thermo Fisher Scientific, Inc., Wilmington, DE, USA). For the detection of miR-663b expression, total RNA was subjected to cDNA synthesis using a TaqMan ${ }^{\mathrm{TM}}$ MicroRNA Reverse Transcription kit (Applied Biosystems; Thermo Fisher Scientific, Inc.), and then qPCR was conducted using a TaqMan ${ }^{\mathrm{TM}}$ MicroRNA Assay kit (Applied Biosystems; Thermo Fisher Scientific, Inc.). The cycling conditions were: $50^{\circ} \mathrm{C}$ for $2 \mathrm{~min}, 95^{\circ} \mathrm{C}$ for $10 \mathrm{~min}, 40$ cycles of denaturation at $95^{\circ} \mathrm{C}$ for $15 \mathrm{sec}$ and annealing/extension at $60^{\circ} \mathrm{C}$ for $60 \mathrm{sec}$. To analyze GAB2 mRNA expression, RT was performed to produce cDNA using a PrimeScript RT Reagent kit (Takara Biotechnology Co., Ltd., Dalian, China), followed by qPCR with a SYBR Premix Ex Taq ${ }^{\mathrm{TM}}$ kit (Takara Biotechnology Co., Ltd.). The thermocycling conditions were: $5 \mathrm{~min}$ at $95^{\circ} \mathrm{C}, 40$ cycles of $95^{\circ} \mathrm{C}$ for $30 \mathrm{sec}$ and $65^{\circ} \mathrm{C}$ for $45 \mathrm{sec}$. U6 small nuclear RNA and GAPDH were used as internal references for miR-663b and GAB2 expression, respectively. All data was calculated by the $2^{-\triangle \Delta C \mathrm{Cq}}$ method (26). The primers were designed as follows: miR-663b, 5'-CGCTAACAGTCTCCAGTC-3' (forward) and 5'-GCGACACCAAACTGGATGA-3' (reverse); U6, 5'-CTC GCTTCGGCAGCACATATACT-3' (forward) and 5'-ACG CTTCACGAATTTGCGTGTC-3' (reverse); GAB2, 5'-CTG AGACTGATAACGGAGAT-3' (forward) and 5'-GAGGTG TTTCTGCTTGAC-3' (reverse); and GAPDH, 5'-CGGAGT CAACGGATTTGGTCGTAT-3' (forward) and 5'-AGCCTT CTCCATGGTGGTGAAGAC-3' (reverse).

CCK-8 assay. Transfected cells were incubated at $37^{\circ} \mathrm{C}$ with $5 \% \mathrm{CO}_{2}$ for $24 \mathrm{~h}$. Next, transfected cells were collected and plated into 96-well plates with a density of $3 \times 10^{3}$ cells per well. CCK-8 assays were performed at $0,1,2$ and 3 days after inoculation to detect cell proliferation, in accordance with the manufacturer's protocol. In detail, $10 \mu \mathrm{l}$ CCK-8 solution (Dojindo Molecular Technologies, Inc., Kumamoto, Japan) was added into each well. Following incubation at $37^{\circ} \mathrm{C}$ for $2 \mathrm{~h}$, the absorbance was measured at a wavelength of $450 \mathrm{~nm}$ using a microplate reader (Bio-Rad Laboratories, Inc., Hercules, CA, USA).

Transwell invasion assay. Transwell plate cell culture chambers (Corning Life Sciences, Cambridge, MA, USA) coated with Matrigel (BD Biosciences, San Jose, CA, USA) were utilized to evaluate invasion capacities of HCC cells. Matrigel coating was performed at $37^{\circ} \mathrm{C}$ for $2 \mathrm{~h}$. Following transfection for $48 \mathrm{~h}$, cells were harvested, washed and diluted into FBS-free DMEM. A total of $1 \times 10^{5}$ transfected cells were placed into the upper compartment of the Transwell plate cell culture chambers, and DMEM containing 20\% FBS was added into the lower compartments. The chambers were then 
Table I. Clinicopathological features of patients with hepatocellular carcinoma.

\begin{tabular}{|c|c|c|c|c|c|c|c|}
\hline Patient no. & Sex & Age & TNM stage & Patient no. & Sex & Age & TNM stage \\
\hline 1 & $\mathrm{~F}$ & 52 & T1N0M0 & 18 & $\mathrm{~F}$ & 63 & T1N0M0 \\
\hline 2 & M & 45 & T2N1M0 & 19 & $\mathrm{~F}$ & 69 & T2N1M0 \\
\hline 3 & $\mathrm{~F}$ & 62 & T2NOM0 & 20 & M & 54 & T2NOMO \\
\hline 4 & $\mathrm{~F}$ & 68 & T1N0M0 & 21 & $\mathrm{~F}$ & 45 & T1N0M0 \\
\hline 5 & M & 58 & T1N0M0 & 22 & M & 60 & T3aN1M0 \\
\hline 6 & M & 69 & T1N0M0 & 23 & M & 66 & T3bN1M0 \\
\hline 7 & M & 58 & T1N0M0 & 24 & M & 71 & TaN1M0 \\
\hline 8 & $\mathrm{M}$ & 67 & T2NOMO & 25 & M & 59 & T2N1M0 \\
\hline 9 & $\mathrm{~F}$ & 53 & T1N0M0 & 26 & $\mathrm{~F}$ & 43 & T1N0M0 \\
\hline 10 & $\mathrm{~F}$ & 70 & T2N1M0 & 27 & M & 67 & T2NOM0 \\
\hline 11 & M & 72 & T1N0M0 & 28 & M & 68 & T1N0M0 \\
\hline 12 & M & 48 & T3aN1M0 & 29 & M & 53 & T3aN1M0 \\
\hline 13 & $\mathrm{~F}$ & 56 & T2N1M0 & 30 & $\mathrm{~F}$ & 64 & TaN0M0 \\
\hline 14 & M & 71 & T1N0M0 & 31 & M & 58 & T3aNOM0 \\
\hline 15 & $\mathrm{M}$ & 63 & T1N0M0 & 32 & $\mathrm{M}$ & 49 & T1N1M0 \\
\hline 16 & M & 49 & T1N0M0 & 33 & M & 47 & T3bN1M0 \\
\hline 17 & $\mathrm{M}$ & 55 & T1N0M0 & 34 & $\mathrm{M}$ & 63 & T1N0M0 \\
\hline
\end{tabular}

F, female; M, male; TNM, tumor node metastasis.

incubated at $37^{\circ} \mathrm{C}$ at $5 \% \mathrm{CO}_{2}$ for $24 \mathrm{~h}$. Non-invasive cells remaining on the upper chamber surface were gently removed using a cotton swab. The invasive cells that had passed through the membranes were fixed with $4 \%$ paraformaldehyde at room temperature for $20 \mathrm{~min}$ and stained with $0.5 \%$ crystal violet at room temperature for $20 \mathrm{~min}$. The number of invasive cells was counted in 5 randomly chosen visuals per chamber under an inverted microscope (IX73; magnification, x200; Olympus Corporation, Tokyo, Japan).

Target prediction for $m i R-663 b$. Bioinformatics analysis was applied to predict the putative targets of miR-663b using TargetScan (http://www.targetscan.org/) and microRNA.org (http://www.microrna.org/). GAB2 was predicted as a major target of miR-663b and was considered for subsequent experimental analysis.

Luciferase reporter assay. The 3'-UTR fragments of GAB2 containing the wild-type (wt) or mutant (mut) miR-663b-binding sequences were synthesized by Shanghai GenePharma Co., Ltd. (Shanghai, China), inserted into the pGL3 luciferase reporter vector (Promega Corporation, Madison, WI,USA) and named as pGL3-wt-GAB2-3'-UTR and pGL3-mut-GAB2-3'-UTR, respectively. Cells were plated into 24 -well plates at a density of $60-70 \%$ confluence $12 \mathrm{~h}$ prior to transfection. Cells were co-transfected with miR-663b mimics (50 pmol) or miR-NC (50 pmol), and pGL3-wt-GAB2-3'-UTR or pGL3-mut-GAB2-3'-UTR, using Lipofectamine ${ }^{\circledR} 2000$ (Invitrogen; Thermo Fisher Scientific, Inc.) based on the manufacturer's instructions. Luciferase activity was measured at $48 \mathrm{~h}$ post-transfection using the Dual-Luciferase reporter system (Promega Corporation). Renilla luciferase activity was used as an internal reference.
Western blot analysis. Western blot analysis was performed to detect GAB2 protein expression. Total protein was isolated from homogenized tissues or cultured cells using cold radioimmunoprecipitation assay buffer, and then was subjected to the detection of protein concentration using a BCA Assay kit (both Nanjing KeyGen Biotech Co., Nanjing, Ltd, China). Protein samples $(20 \mu \mathrm{g})$ were separated on a $10 \%$ SDS-PAGE and transferred onto polyvinylidene difluoride membranes (EMD Millipore, Billerica, MA, USA). The membranes were then blocked at room temperature for $2 \mathrm{~h}$ with $5 \%$ skimmed milk diluted in TBS $/ 0.1 \%$ Tween (TBST) and incubated overnight at $4^{\circ} \mathrm{C}$ with primary antibodies. Following extensive washing with TBST, the membranes were additionally probed with the goat anti-rabbit horseradish peroxidase-conjugated secondary antibody (cat. no. ab205718; 1:5,000 dilution; Abcam, Cambridge, UK) for $2 \mathrm{~h}$ at room temperature, followed by visualization of the protein bands using an enhanced chemiluminescence reagent (Bio-Rad Laboratories, Inc.). The primary antibodies used in the present study included rabbit anti-human GAB2 antibody (cat. no. ab108423; 1:1,000 dilution; Abcam) and rabbit anti-human GAPDH antibody (cat. no. ab128915; 1:1,000 dilution; Abcam). GAPDH was used as a loading control.

Statistical analysis. All data are presented as the means \pm standard deviation, and were analyzed using the SPSS 21.0 software (IBM Corp., Armonk, NY, USA). The differences between two groups were examined using Student's t-test or Wilcoxon signed-rank test, and differences between three or more groups were analyzed using one-way analysis of variance (ANOVA). The Student-Newman-Keuls test was employed as a post-hoc test following ANOVA. The association between miR-663b and GAB2 mRNA levels in HCC tissues was determined 
using Spearman's correlation analysis. $\mathrm{P}<0.05$ was considered to indicate a statistically significant difference.

\section{Results}

miR-663b is decreased in HCC tissues and cell lines. To detect the expression status of miR-663b in HCC, miR-663b expression in $\mathrm{HCC}$ was first determined by initially comparing the levels of miR-663b in 34 pairs of HCC tissues and adjacent normal tissues. Results from RT-qPCR analysis revealed that the expression levels of miR-663b were decreased in HCC tissues compared with that in adjacent normal tissues (Fig. 1A; $\mathrm{P}<0.05)$. miR-663b expression was then additionally measured in 2 human HCC Huh7 and Hep3B cell lines, and an immortalized normal human liver epithelial L-O2 cell line. Significantly decreased expression levels of miR-663b were observed in the $2 \mathrm{HCC}$ cell lines compared with that in L-O2 (Fig. 1B; $\mathrm{P}<0.05$ ). These results suggest that the decreased miR-663b expression may be associated with the progression and development of HCC.

miR-663b overexpression suppresses HCC cell proliferation and invasion. To examine the biological roles of miR-663b in HCC, Huh7 and Hep3B cells were treated with miR-663 mimics to increase its endogenous expression (Fig. 2A; $\mathrm{P}<0.05)$. The effect of miR-663b overexpression on HCC cell proliferation was determined by CCK-8 assay. The results indicated that ectopic miR-663b expression significantly inhibited the proliferation of Huh7 and Hep3B cells (Fig. 2B; P<0.05). In addition, a Transwell invasion assay was applied to detect the invasion ability of Huh7 and Hep3B cells transfected with miR-663b mimics or miR-NC. The invasion ability of Huh7 and Hep3B cells was markedly decreased following treatment with miR-663b mimics (Fig. 2C; $\mathrm{P}<0.05$ ). Taken together, these results suggested that miR-663b may serve as a tumor suppressor in HCC.

miR-663b inhibits GAB2 expression in HCC cells by binding to its 3'-UTR. To explore the fundamental mechanisms of miR-663b in HCC cells, bioinformatics analysis was used to predict the potential targets of miR-663b. A total of 135 genes were predicted as candidates of miR-663b, including GAB2, tumor suppressor candidate 2 (TUSC2), sex-determining region Y-box 12, chromodomain-helicase-DNA-binding protein 5, cyclin D2 and transcription factor forkhead box A2. GAB2 was predicted as a major target of miR-663b (Fig. 3A) and was considered for additional experimental analysis as GAB2 has been demonstrated to be closely associated with HCC oncogenesis and development (27-29). To affirm this prediction, a luciferase reporter assay was performed to clarify whether miR-663b may directly interact with the 3'-UTR of GAB2. Fig. 3B indicated that miR-663b overexpression suppressed the luciferase activity of the reporter plasmid with wt 3'-UTR of GAB2 in Huh7 and Hep3B cells $(\mathrm{P}<0.05)$; however, the luciferase activity of the reporter plasmid carrying the mut 3'-UTR of GAB2 was unaffected. In order to additionally confirm that GAB2 was a direct target for miR-663b, RT-qPCR and western blot analysis were performed to detect GAB2 mRNA and protein levels in Huh7 and Hep3B cells following transfection with miR-663b or miR-NC. The
A

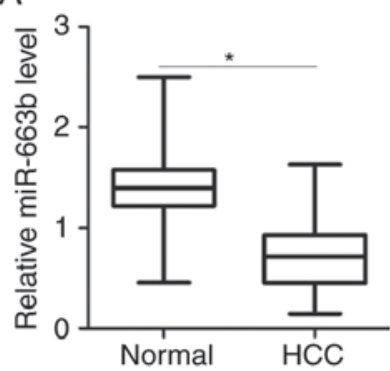

B

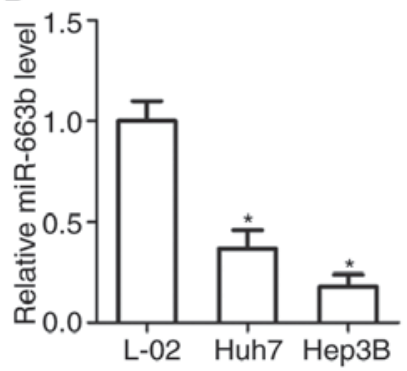

Figure 1. miR-663b expression is downregulated in HCC tissues and cell lines. (A) Reverse transcription-quantitative polymerase chain reaction analysis was used to detect miR-663b expression in 34 pairs of HCC tissues and adjacent normal tissues. $\mathrm{P}<0.05$ vs. adjacent normal tissues. (B) Comparison of the differences in miR-663b expression between 2 human HCC Huh7 and Hep3B cell lines and an immortalized normal human liver epithelial L-O2 cell line. ${ }^{*} \mathrm{P}<0.05$ vs. L-O2. miR, microRNA; HCC, hepatocellular carcinoma.

results demonstrated that mRNA (Fig. 3C; $\mathrm{P}<0.05$ ) and protein (Fig. 3D; P<0.05) levels of GAB2 in Huh7 and Hep3B cells were markedly downregulated by miR-663b overexpression. Therefore, GAB2 is a direct target of miR-663b in HCC cells.

miR-663b expression is inversely associated with GAB2 in HCC tissues. To additionally investigate the association between miR-663b and GAB2 in HCC, GAB2 expression was measured in 34 pairs of $\mathrm{HCC}$ tissues and adjacent normal tissues. The data from the RT-qPCR analysis indicated that GAB2 mRNA levels were upregulated in HCC tissues compared with that in adjacent normal tissues (Fig. 4A; $\mathrm{P}<0.05)$. Concomitantly, western blot analysis revealed that the expression level of GAB2 protein was significantly increased in HCC tissues compared with that in adjacent normal tissues (Fig. 4B; P<0.05). Furthermore, Spearman's correlation analysis revealed an inverse correlation between miR-663b and GAB2 mRNA expression in HCC tissues (Fig. 4C; $r=-0.5904$; $\mathrm{P}=0.0002$ ). These results suggest that the upregulation of GAB2 in HCC tissues was, at least partly, caused by miR-663b downregulation.

GAB2overexpressionrescuestheinhibitoryeffectsofmiR- $663 b$ overexpression on HCC cell proliferation and invasion. A series of rescue experiments were performed to determine whether GAB2 overexpression may reverse cell proliferation and invasion suppression caused by miR-663b upregulation in Huh7 and Hep3B cells. Therefore, pcDNA3.1-GAB2 or empty pcDNA3.1 plasmids were transfected into Huh7 and Hep3B cells, and western blot analysis was performed to detect GAB2 protein expression. GAB2 protein levels were significantly increased in pcDNA3.1-GAB2-transfected Huh7 and Hep3B cells compared with that in cells transfected with pcDNA3.1 (Fig. 5A; P<0.05). Western blot analysis also identified that the downregulation of GAB2 protein induced by miR-663b overexpression was restored in Huh7 and Hep3B cells by co-transfecting with pcDNA3.1-GAB2 (Fig. 5B and C; $\mathrm{P}<0.05)$. Furthermore, CCK-8 and Transwell invasion assays demonstrated that GAB2 overexpression partially rescued the inhibitory effects of miR-663b on the proliferation 
A
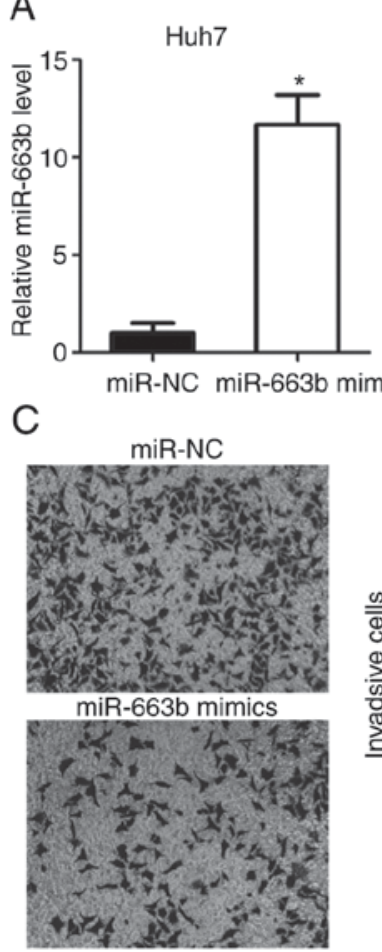

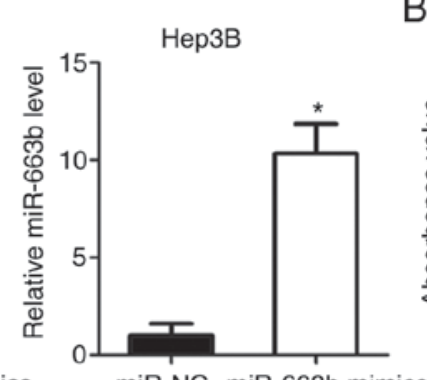

miR-NC miR-663b mimics
B

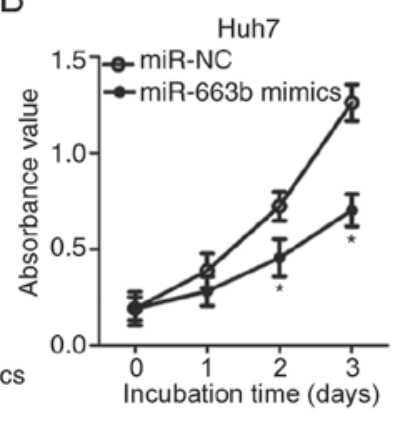

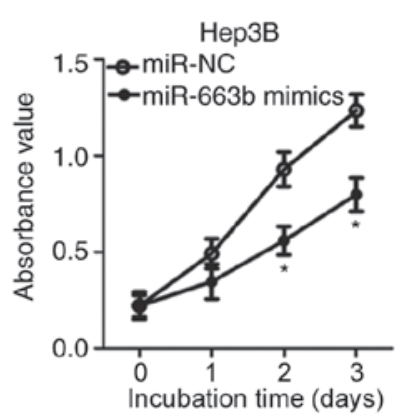

miR-NC
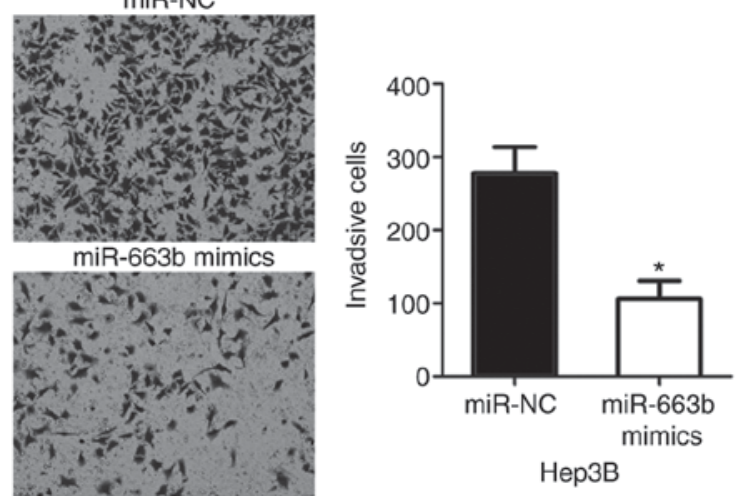

Figure 2. Overexpression of miR-663b restricts the proliferation and invasion of Huh7 and Hep3B cells. (A) Reverse transcription-quantitative polymerase chain reaction was performed to measure miR-663b expression in Huh7 and Hep3B cells transfected with miR-663b mimics or miR-NC. ${ }^{*} \mathrm{P}<0.05$ vs. miR-NC. (B) A CCK-8 assay was utilized to determine proliferation of Huh7 and Hep3B cells treated with miR-663b mimics or miR-NC. * $<<0.05$ vs. miR-NC. (C) Invasion capacity was examined by a Transwell invasion assay in Huh7 and Hep3B cells that were transfected with miR-663b mimics or miR-NC (magnification, $\mathrm{x} 200$ ). ${ }^{*} \mathrm{P}<0.05$ vs. miR-NC. miR, microRNA; NC, negative control.

A

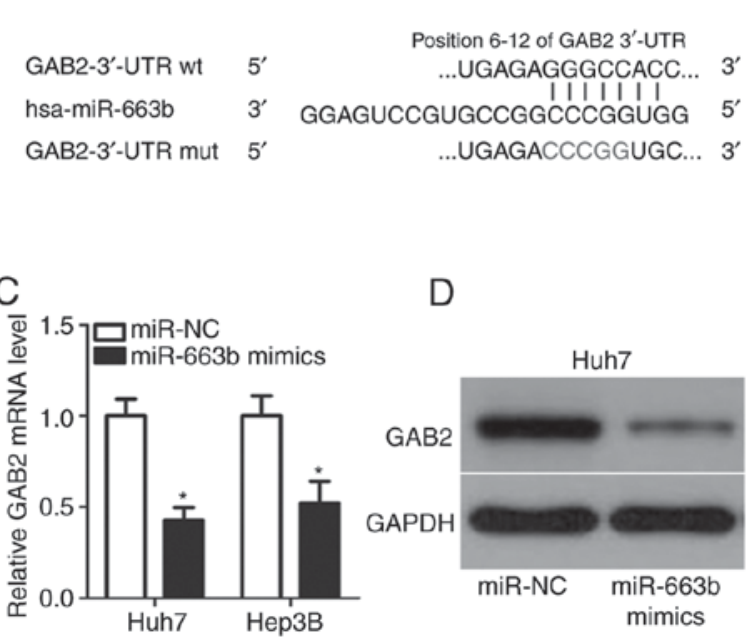

B
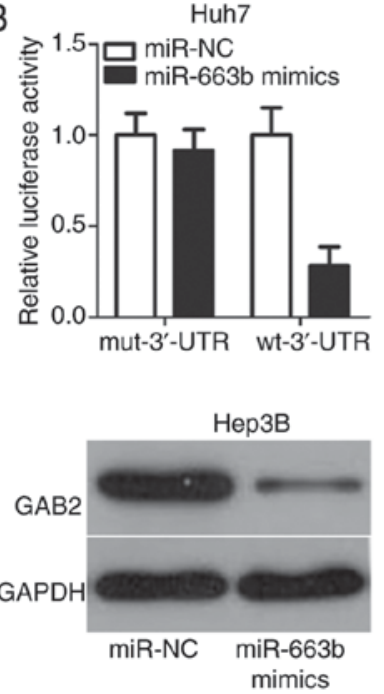
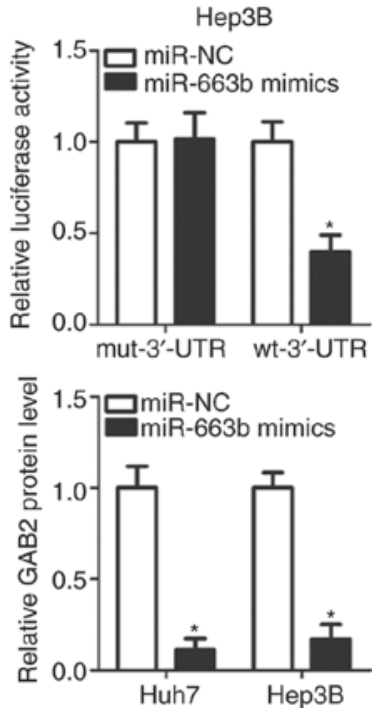

Figure 3. GAB2 is a direct target gene of miR-663b in hepatocellular carcinoma. (A) Bioinformatics analysis predicted that GAB2 may be a potential target of miR-663b. The 3'-UTR of GAB2 harboring the predictive wt or mut miR-663b binding site was cloned into the pGL3 luciferase reporter vector. (B) Luciferase activity was detected in Huh7 and Hep3B cells co-transfected with pGL3-wt-GAB2-3'-UTR or pGL3-mut-GAB2-3'-UTR and miR-663b mimics or miR-NC. Renilla luciferase activity was used as an internal reference. ${ }^{*} \mathrm{P}<0.05$ vs. miR-NC. (C) Reverse transcription-quantitative polymerase chain reaction and (D) western blot analysis were employed to measure GAB2 mRNA and protein expression levels in Huh7 and Hep3B cells treated with miR-663b mimics or miR-NC. " $\mathrm{P}<0.05$ vs. miR-NC. GAB2, Grb2-associated binding 2; miR, microRNA; hsa, Homo sapiens; UTR, untranslated region; wt, wild type; mut, mutant; NC, negative control.

(Fig. 5D; P<0.05) and invasion (Fig. 5E; P<0.05) of Huh7 and Hep3B cells. These data confirmed that miR-663b may serve inhibitory roles in HCC, at least partly, by decreasing GAB2 expression.

\section{Discussion}

Previous studies have demonstrated that numerous tumor-specific miRNAs are upregulated or downregulated in 
A

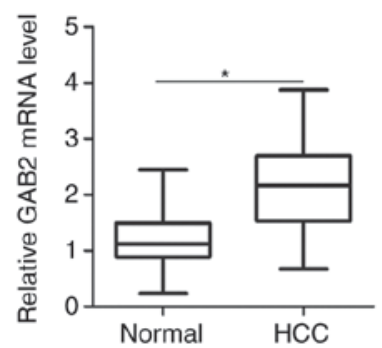

B

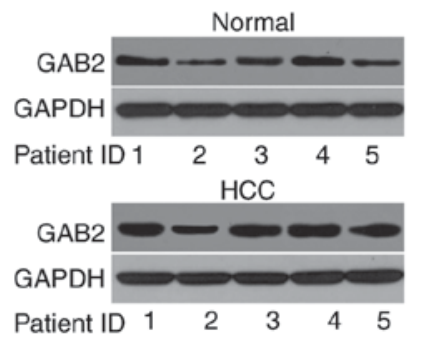

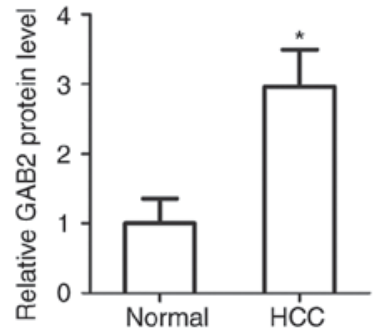

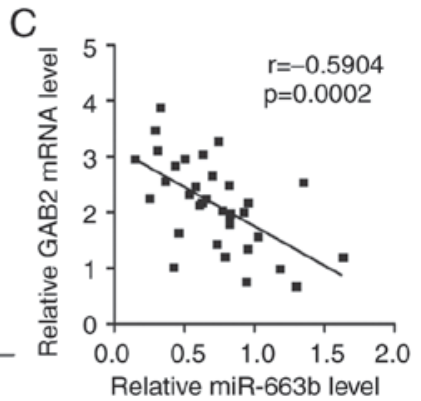

Figure 4. GAB2 is upregulated in HCC tissues and upregulation of GAB2 is inversely correlated with miR-663b expression. (A) Reverse transcription-quantitative polymerase chain reaction analysis was used to detect GAB2 mRNA expression in 34 pairs of HCC tissues and adjacent normal tissues. "P<0.05 vs. adjacent normal tissues. (B) The protein expression of GAB2 was determined in HCC tissues and adjacent normal tissues via western blot analysis. "P<0.05 vs. adjacent normal tissues. (C) The mRNA level of GAB2 was negatively correlated with miR-663b expression in HCC tissues by Spearman's correlation analysis $\mathrm{r}=-0.5904, \mathrm{P}=0.0002$. HCC, hepatocellular carcinoma; GAB2, Grb2-associated binding 2; miR, microRNA.
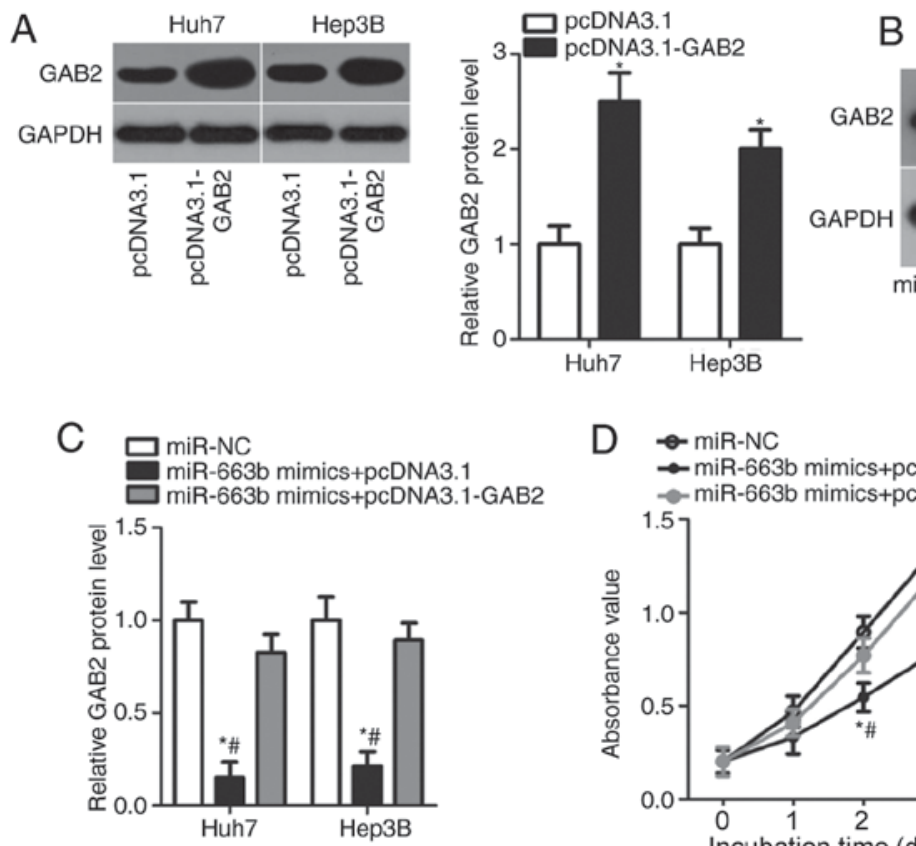

B

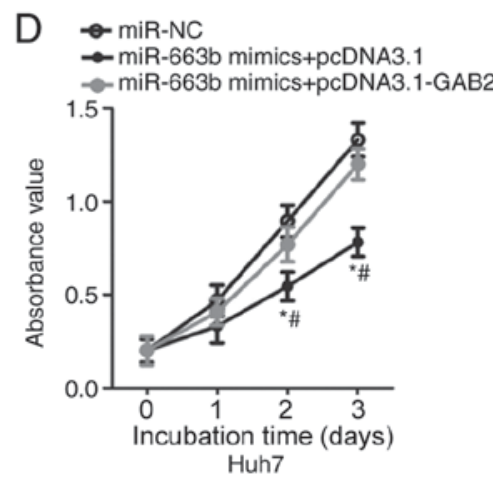

Huh7
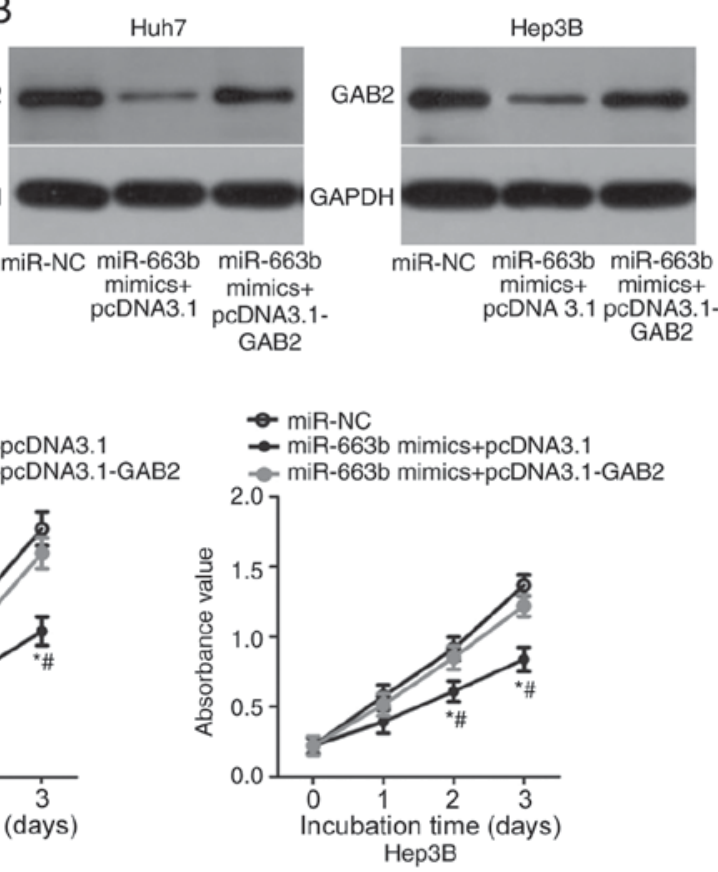

Нер3B

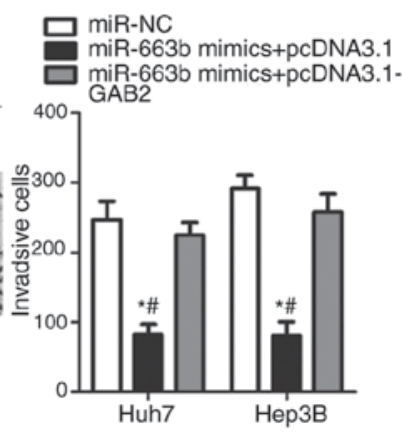

$\mathrm{E}$
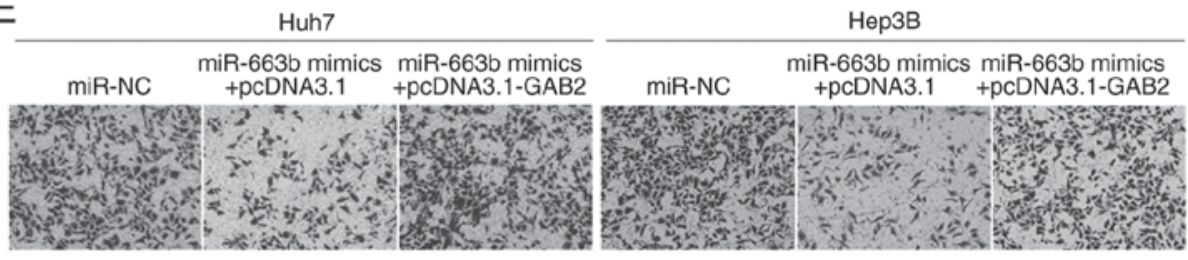

Figure 5. Upregulation of GAB2 partially abolishes miR-663b-mediated inhibition of hepatocellular carcinoma cell proliferation and invasion. (A) Western blot analysis was performed to determine GAB2 protein expression in Huh7 and Hep3B cells following transfection with pcDNA3.1-GAB2 or empty pcDNA3.1 plasmids. * $\mathrm{P}<0.05$ vs. pcDNA3.1. (B) miR-663b mimics, together with pcDNA3.1-GAB2 or pcDNA3.1, were introduced into Huh7 and Hep3B cells. Western blot analysis was performed to detect GAB2 protein expression at $72 \mathrm{~h}$ post-transfection. (C) Densitometric analysis of western blot analysis data. ${ }^{*} \mathrm{P}<0.05$ vs. miR-NC; ${ }^{*} \mathrm{P}<0.05$ vs. miR-663b mimics+pcDNA3.1-GAB2. (D) Cell proliferation and (E) invasion rates were detected using CCK-8 and Transwell

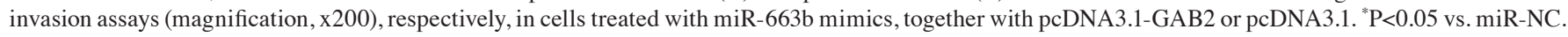
${ }^{\#} \mathrm{P}<0.05$ vs. miR-663b mimics+pcDNA3.1-GAB2. GAB2, Grb2-associated binding 2; miR, microRNA; NC, negative control.

$\mathrm{HCC}$, and that their dysregulation is implicated in the HCC occurrence and development (30-32). Therefore, investigation of crucial miRNAs involved in HCC oncogenesis and progression may provide novel insights into the therapy of patients with this malignant tumor. The present study demonstrated that miR-663b expression was significantly 
downregulated in HCC tissues and cell lines. Exogenous overexpression of miR-663b impeded the proliferation and invasion of HCC cells. Additionally, GAB2 was demonstrated to be a direct target gene of miR-663b in HCC cells. Furthermore, it was identified that miR-663b expression was negatively correlated with GAB2 expression in HCC tissues. Finally, restoration of GAB2 expression partially abrogated the tumor suppressive roles of miR-663b in HCC cells. All these results demonstrated that miR-663b is downregulated in $\mathrm{HCC}$, and inhibits the proliferation and invasion of HCC cells by directly targeting GAB2.

miR-663b dysregulation is observed in several types of human malignancies. For example, miR-663b is upregulated in nasopharyngeal carcinoma tissues and cell lines (21). High miR-663b expression is markedly associated with clinical stage and lymph node metastasis of patients with nasopharyngeal carcinoma (21). Expression of miR-663b was increased in the plasma of patients with bladder cancer (22). miR-663b level was suggested to be an effective circulating biomarker for the diagnosis of bladder cancer (22). miR-663b is also overexpressed in osteosarcoma (23) and endometrial cancer (24) tissues. Patients with endometrial cancer with high miR-663b levels exhibited poorer prognosis compared with those patients with low miR-663b levels (24). However, miR-663b is downregulated in pancreatic cancer tissues and cell lines (33). These contrary data indicated that the expression pattern of miR-663b in human cancer is tissue-specific. Therefore, miR-663b may be a promising biomarker for the diagnosis of these human cancer types.

Aberrantly expressed miR-663b serves oncogenic roles in the progression of multiple human cancer types. For example, miR-663b overexpression promotes cell growth and metastasis of nasopharyngeal carcinoma (21). Shu et al (23) demonstrated that inhibition of miR-663b suppresses the proliferation and induced apoptosis of osteosarcoma cells. Wang et al (24) identified that miR-663b re-expression promotes cell viability and repressed cell apoptosis in endometrial cancer. Nevertheless, miR-663b serves tumor-suppressing roles in pancreatic cancer via affecting cell proliferation, colony formation, migration, invasion and apoptosis in vitro and tumor growth in vivo (33). These conflicting results demonstrated that the biological roles of miR-663b in the tumorigenesis and tumor development exhibit tissue specificity. In general, miR-663b may be developed as a prospective therapeutic cancer target for the treatment of patients with these specific cancer types.

Multiple genes have been confirmed to be the direct target genes of miR-663b, including TUSC2 in nasopharyngeal carcinoma (21), tumor protein P73 in osteosarcoma (23), apoptosis facilitator $\mathrm{Bcl}-2$-like protein 14 in endometrial cancer (24) and insulin-like growth factor 2 in pancreatic cancer (33). In the present study, GAB2, a member of the mammalian Gab scaffolding/adapter family, was validated as a direct and functional downstream target of miR-663b in $\mathrm{HCC}$; its expression was upregulated in HCC tissues and a subset of HCC cell lines compared with normal tissues and cell lines. High GAB2 expression exhibits a significant correlation with histological grade and tumor size in patients with HCC $(27,28)$. Patients with HCC with high GAB2 expression exhibit poorer prognosis compared with those patients with low GAB2 levels (27). Kaplan-Meier survival curves identified GAB2 expression level as an independent prognostic marker for predicting overall survival of patients with HCC (28). In addition, GAB2 serves as an oncogene in HCC carcinogenesis and cancer progression through regulating a variety of biological behaviors, including cell proliferation, cycle, apoptosis, migration and invasion in vitro and tumor growth in vivo $(28,29)$. On the basis of the results of the present study, miR-663b and its target GAB2 may be potential therapeutic targets for patients with $\mathrm{HCC}$.

In conclusion, the results of the present study demonstrated that miR-663b is downregulated in HCC tissues and cell lines. The restoration of miR-663b expression inhibited cell proliferation and invasion in HCC through directly targeting and inhibiting GAB2. These results suggested the key roles of miR-663b in HCC progression, in the diagnosis and therapy of patients with HCC. However, the present study did not investigate the effects of miR-663b in HCC cell apoptosis in vitro, or in vivo tumor growth and metastasis. In addition, the association between GAB2 expression and histological grades in patients with HCC was not described. These are limitations of the present study, and will be resolved in the future.

\section{Acknowledgements}

Not applicable.

\section{Funding}

The present study was supported by the Special Research Funds for Discipline Construction of High Level University Construction (grant no. 2013SXTS02).

\section{Availability of data and materials}

The datasets used and/or analyzed during the present study are available from the corresponding author on reasonable request.

\section{Authors' contributions}

LG and JY designed the study. LG and BL performed the CCK-8 assay and reverse transcription-quantitative polymerase chain reaction. MM conducted the Transwell invasion and luciferase reporter assays. Western blot analysis and statistical analysis were performed by JY and JJ. All authors read and approved the final draft.

\section{Ethics approval and consent to participate}

The present study was approved by the Research Ethics Committee of Affiliated Hospital of Yan'an University, and was performed in accordance with the Declaration of Helsinki and the guidelines of the Ethics Committee of Affiliated Hospital of Yan'an University. Written informed consent was obtained from all patients for the use of their clinical tissues.

\section{Patient consent for publication}

All patients enrolled in this study provided written informed consent for the publication of any associated data. 


\section{Competing interests}

The authors declare that they have no competing interests.

\section{References}

1. Siegel RL, Miller KD and Jemal A: Cancer statistics, 2016. CA Cancer J Clin 66: 7-30, 2016.

2. Global Burden of Disease Cancer Collaboration, Fitzmaurice C, Dicker D, Pain A, Hamavid H, Moradi-Lakeh M, MacIntyre MF, Allen C, Hansen G, Woodbrook R, et al: The global burden of cancer 2013. JAMA Oncol 1: 505-527, 2015.

3. Cabrera R and Nelson DR: Review article: The management of hepatocellular carcinoma. Aliment Pharmacol Ther 31: 461-476, 2010 .

4. Marrero JA and Welling T: Modern diagnosis and management of hepatocellular carcinoma. Clin Liver Dis 13: 233-247, 2009.

5. Forner A, Hessheimer AJ, Isabel Real M and Bruix J: Treatment of hepatocellular carcinoma. Crit Rev Oncol Hematol 60: 89-98, 2006.

6. Ishizawa T, Hasegawa K, Aoki T, Takahashi M, Inoue Y, Sano K, Imamura H, Sugawara Y, Kokudo N and Makuuchi M: Neither multiple tumors nor portal hypertension are surgical contraindications for hepatocellular carcinoma. Gastroenterology 134: 1908-1916, 2008.

7. Forner A, Llovet JM and Bruix J: Hepatocellular carcinoma. Lancet 379: 1245-1255, 2012.

8. Yu MC and Yuan JM: Environmental factors and risk for hepatocellular carcinoma. Gastroenterology 127 (5 Suppl 1): S72-S78, 2004.

9. Borchert GM, Lanier W and Davidson BL: RNA polymerase III transcribes human microRNAs. Nat Struct Mol Biol 13: 1097-1101, 2006.

10. Bartel DP: MicroRNAs: Genomics, biogenesis, mechanism, and function. Cell 116: 281-297, 2004.

11. Klingenberg M,Matsuda A,Diederichs S and Patel T: Non-coding RNA in hepatocellular carcinoma: Mechanisms, biomarkers and therapeutic targets. J Hepatol 67: 603-618, 2017.

12. Weidle UH, Birzele F, Kollmorgen G and Nopora A: Potential microRNA-related targets for therapeutic intervention with ovarian cancer metastasis. Cancer Genomics Proteomics 15: $1-15,2018$.

13. Celano M, Rosignolo F, Maggisano V, Pecce V, Iannone M, Russo D and Bulotta S: MicroRNAs as Biomarkers in Thyroid Carcinoma. Int J Genomics 2017: 6496570, 2017.

14. Irmak-Yazicioglu MB: Mechanisms of microRNA deregulation and microRNA targets in gastric cancer. Oncol Res Treat 39: 136-139, 2016.

15. Zhang Y, Yang L, Wang S, Liu Z and Xiu M: MiR-29a suppresses cell proliferation by targeting SIRT1 in hepatocellular carcinoma. Cancer Biomark 22: 151-159, 2018.

16. Li H, Wang H and Ren Z: MicroRNA-214-5p inhibits the invasion and migration of hepatocellular carcinoma cells by targeting Wiskott-Aldrich syndrome like. Cell Physiol Biochem 46: 757-764, 2018.

17. Wu M, Huang C, Huang $X$, Liang $R$, Feng $Y$ and Luo $X$ : MicroRNA-144-3p suppresses tumor growth and angiogenesis by targeting SGK3 in hepatocellular carcinoma. Oncol Rep 38: 2173-2181, 2017.
18. Hayes $\mathrm{CN}$ and Chayama K: MicroRNAs as biomarkers for liver disease and hepatocellular carcinoma. Int J Mol Sci 17: 280, 2016.

19. Anwar SL and Lehmann U: MicroRNAs: Emerging Novel Clinical Biomarkers for Hepatocellular Carcinomas. J Clin Med 4: 1631-1650, 2015.

20. Liu Z, Wang Y, Dou C, Sun L, Li Q, Wang L, Xu Q, Yang W, Liu Q and Tu K: MicroRNA-1468 promotes tumor progression by activating PPAR-gamma-mediated AKT signaling in human hepatocellular carcinoma. J Exp Clin Cancer Res 37: 49, 2018.

21. Liang S, Zhang N, Deng Y, Chen L, Zhang Y, Zheng Z, Luo W, Lv Z, Li S and Xu T: miR-663b promotes tumor cell proliferation, migration and invasion in nasopharyngeal carcinoma through targeting TUSC2. Exp Ther Med 14: 1095-1103, 2017.

22. Du M, Shi D, Yuan L, Li P, Chu H, Qin C, Yin C, Zhang Z and Wang M: Circulating miR-497 and miR-663b in plasma are potential novel biomarkers for bladder cancer. Sci Rep 5: 10437 , 2015.

23. Shu Y, Ye W, Gu YL and Sun P: Blockade of miR-663b inhibits cell proliferation and induces apoptosis in osteosarcoma via regulating TP73 expression. Bratisl Lek Listy 119: 41-46, 2018.

24. Wang YL, Shen Y, Xu JP, Han K, Zhou Y, Yang S, Yin JY, Min DL and Hu HY: Pterostilbene suppresses human endometrial cancer cells in vitro by down-regulating miR-663b. Acta Pharmacol Sin 38: 1394-1400, 2017.

25. Faria SC, Szklaruk J, Kaseb AO, Hassabo HM and Elsayes KM: TNM/Okuda/Barcelona/UNOS/clip international multidisciplinary classification of hepatocellular carcinoma: Concepts, perspectives, and radiologic implications. Abdom Imaging 39: 1070-1087, 2014

26. Livak KJ and Schmittgen TD: Analysis of relative gene expression data using real-time quantitative PCR and the 2(-Delta Delta C(T)) method. Methods 25: 402-408, 2001.

27. Hu X, He B, Zhou L, Xie H and Zheng S: Expression pattern and clinical significance of Gab2 protein in hepatocellular carcinoma. Clin Lab 62: 1087-1092, 2016.

28. Chen Y, Liu Q, Wu M, Li M, Ding H, Shan X, Liu J, Tao T, Ni R and Chen $\mathrm{X}$ : GAB2 promotes cell proliferation by activating the ERK signaling pathway in hepatocellular carcinoma. Tumour Biol 37: 11763-11773, 2016.

29. Cheng J, Zhong Y, Chen S, Sun Y, Huang L, Kang Y, Chen B, Chen G, Wang F, Tian Y, et al: Gab2 mediates hepatocellular carcinogenesis by integrating multiple signaling pathways. FASEB J 31: 5530-5542, 2017.

30. Zhang T, Liu W, Meng W, Zhao H, Yang Q, Gu SJ, Xiao CC, Jia CC and Fu BS: Downregulation of miR-542-3p promotes cancer metastasis through activating TGF-beta/Smad signaling in hepatocellular carcinoma. Onco Targets Ther 11: 1929-1939, 2018.

31. Cui H, Song R, Wu J, Wang W, Chen X and Yin J: MicroRNA-337 regulates the $\mathrm{PI} 3 \mathrm{~K} / \mathrm{AKT}$ and $\mathrm{Wnt} / \beta$-catenin signaling pathways to inhibit hepatocellular carcinoma progression by targeting high-mobility group AT-hook 2. Am J Cancer Res 8: 405-421, 2018.

32. Morishita A and Tsutomu M: MicroRNAs as possible biomarkers for hepatocellular carcinoma. Hepatol Res 48: 499-501, 2018.

33. Cai H, An Y, Chen X, Sun D, Chen T, Peng Y, Zhu F, Jiang Y and He X: Epigenetic inhibition of miR-663b by long non-coding RNA HOTAIR promotes pancreatic cancer cell proliferation via up-regulation of insulin-like growth factor 2 . Oncotarget 7: 86857-86870, 2016. 